\title{
A protocol for a single-center, single- blinded randomized-controlled trial investigating volar plating versus conservative treatment of unstable distal radius fractures in patients older than 65 years
}

Jonas Pedersen ${ }^{1,3}$, Simon Oksbjerre Mortensen ${ }^{1}$, Jan Duedal Rölfing ${ }^{2,3}$ and Rikke Thorninger ${ }^{1 *}$

\begin{abstract}
Background: Distal radius fractures (DRF) are very common in elderly patients, who present at the Emergency Department. Surgical treatment with open reduction and internal fixation using volar locking plates is widely prevalent despite the lack of evidence proving its superiority to conservative treatment with closed reduction and plaster immobilization. The purpose of this study is to investigate whether conservative treatment is superior to volar plating in terms of number of complications and results in a comparable or superior functional outcome in patients $\geq 65$ years.
\end{abstract}

Methods: In this single-center, single-blinded randomized-controlled trial, patients $\geq 65$ years with distal radius fractures will be invited to participate. A total of 50 patients per treatment arm is required to provide $80 \%$ statistical power at a $5 \%$ alpha level assuming a difference of $20 \%$ in complication rate between operatively and conservatively treated patients. Primary outcome measures will be complication rate, Quick DASH score (Quick Disabilities of the Arm, Shoulder and Hand), PRWE (Patient rated Wrist evaluation), and range of motion of the wrist. Secondary outcome measures will be grip strength, pinch gauge, pain, use of pain medication EQ5D score (European Quality of life - 5 dimensions), standardized radiographs. One year of follow-up is planned with data collection at the day of injury, after 2 weeks, after 5 weeks, after 6 months, and after 12 months. An intention-totreat and per-protocol analysis will be performed.

Discussion: This prospective trial helps to clarify the best treatment strategy for displaced DRF patients $\geq 65$ years.

Trial registration: This trial is approved by the Danish Scientific Ethical Committee (ID: 1-10-72-420-17) and registered at Clinicaltrials.gov (Trial registration number NCT03716661).

Keywords: Distal radius fractures, Volar plating, Conservative, Complications, Functional outcome, Elderly

\footnotetext{
* Correspondence: rikkthor@rm.dk

${ }^{1}$ Department of Orthopedics, Regional Hospital Randers, Skovlyvej 15,

DK-8930 Randers, Denmark

Full list of author information is available at the end of the article
}

(c) The Author(s). 2019 Open Access This article is distributed under the terms of the Creative Commons Attribution 4.0 International License (http://creativecommons.org/licenses/by/4.0/), which permits unrestricted use, distribution, and reproduction in any medium, provided you give appropriate credit to the original author(s) and the source, provide a link to the Creative Commons license, and indicate if changes were made. The Creative Commons Public Domain Dedication waiver (http://creativecommons.org/publicdomain/zero/1.0/) applies to the data made available in this article, unless otherwise stated. 


\section{Background}

Distal radius fractures (DRF) account for $18 \%$ of all fractures in the elderly $\geq 65$ years of age [1]. The estimated lifetime risk for DRF is $15 \%$ for females and $2 \%$ for males [2]. The incidence rate is $190-200$ per 100,000 person-years [3]. DRF is associated with osteoporosis, hence the agerelated incidence rate increases almost 3 -fold from the age of 60 to 99 in women $[1,4,5]$. In Europe, the proportion of the elderly population is estimated to increase by $56 \%$ in men and by $41 \%$ in women until 2035 [6, 7]. Consequently, the need to clarify the best treatment strategy for DRF in elderly is evident.

In recent years, there has been a trend to treat DRF patients that require surgical treatment with an open reduction and internal fixation (ORIF) using a volar locking plate. The Danish Health Authority stipulates in the National Clinical Guidelines (NCG) regarding the treatment of low-energy DRF [8] to volar plate fractures that fulfill the following radiologic criteria after attempted closed reduction:

$->10^{\circ}$ dorsal tilt of the radius in relation to perpendicular to the longitudinal axis of radius

- $>2 \mathrm{~mm}$ articular step-off

- $>2$ mm ulnar variance

- incongruence of the distal radioulnar joint

- substantial dorsal comminution indicating gross instability

If one or more of these criteria are met, ORIF most often utilizing a volar locking plate is advised regardless of the patient's age. The guideline also highlights that conservative management should be considered in patients with low functional demands.

Notably, the guideline does not include recommendations for high-energy, open fractures nor grossly instable fractures: volarly displaced (Smith), radial styloid (Cheuffeur) or articular rim (Barton) fractures. However, most of these fractures are also treated with volar locking plates in Denmark.

The radiological NCG criteria rely on clinical observations only and have not been systematically evaluated prospectively. Furthermore, the reliability of the radiological criteria has been questioned [9]. Therefore, The Danish Health Authority evaluates that the recommendations for treating DRF primarily are based on low quality evidence and must be considered as "good practice"-guidelines.

Volar plating of DRF may harm patients. Complication rates of up to $33 \%$ have been reported in surgically treated DRF patients [10-14]. Our own estimation of the complication rate after volar plating of DRF is $14.6 \%$ [95\% CI $11.8-17.7 \%$ ] in a retrospective cohort of 595 patients with 3.2 years follow-up [3]. This high complication rate is not insignificant for the patients. Neurologic disturbances, tendon irritation and rupture, infection, etc. often lead either to a re-operation or an increase in out-patient visits and may result in permanent morbidity and impaired function [15].

Furthermore, volar plating improves the early functional recovery, but long-term functional results are similar with other treatment modalities in patients $\geq 65$ years [16]. However, volar plating is still the gold standard in the treatment of DRF in adults regardless of age.

An increase in complications with no clinically significant difference between the functional outcome of operatively and conservatively treated patients was demonstrated $[16,17]$. Furthermore, the patients treated operatively had a higher complications rate than the conservatively treated patients [17]. In addition, patients with DRF treated nonoperatively have shown to have less pain and better or equal wrist function after a 1 year follow-up than those treated surgically [18]. A Danish review of operatively treated patients suggest a more restrictive choice of treatment for DRF amongst the elderly than the NCG stipulate [2]. All things considered, no existing evidence proves the benefit of treating DRF operatively in the elderly.

Here, we question whether the potential benefit of volar plating, namely an earlier functional recovery outweighs the risk of encountering a complication. Especially in the light of retired patients ( $\geq 65$ years), where return to work is not a burning issue, it seems worthwhile to investigate this issue both in the interest of the patients and society.

\section{Research hypothesis}

Patients above 65 years of age, who sustain a DRF that fulfill the national radiologic criteria for operative treatment will experience fewer complications when treated with dorsal plaster cast immobilization only than when operated using a volar locking plate.

Meanwhile, the secondary outcome measures will be comparable and below the clinically relevant difference - e.g. a mean difference in the Danish version of the Quick Disabilities of the Arm, Shoulder and Hand (Quick DASH) below 16-20 point [19-22].

\section{Methods/design \\ Study design}

A prospective single-center, single-blinded randomizedcontrolled superiority trial with two parallel treatment arms and a third control arm (Fig. 1);

Arm 1: volar plating, 2-weeks dorsal plaster cast followed by 3 -weeks orthosis immobilization with a single hand therapeutic instruction; 


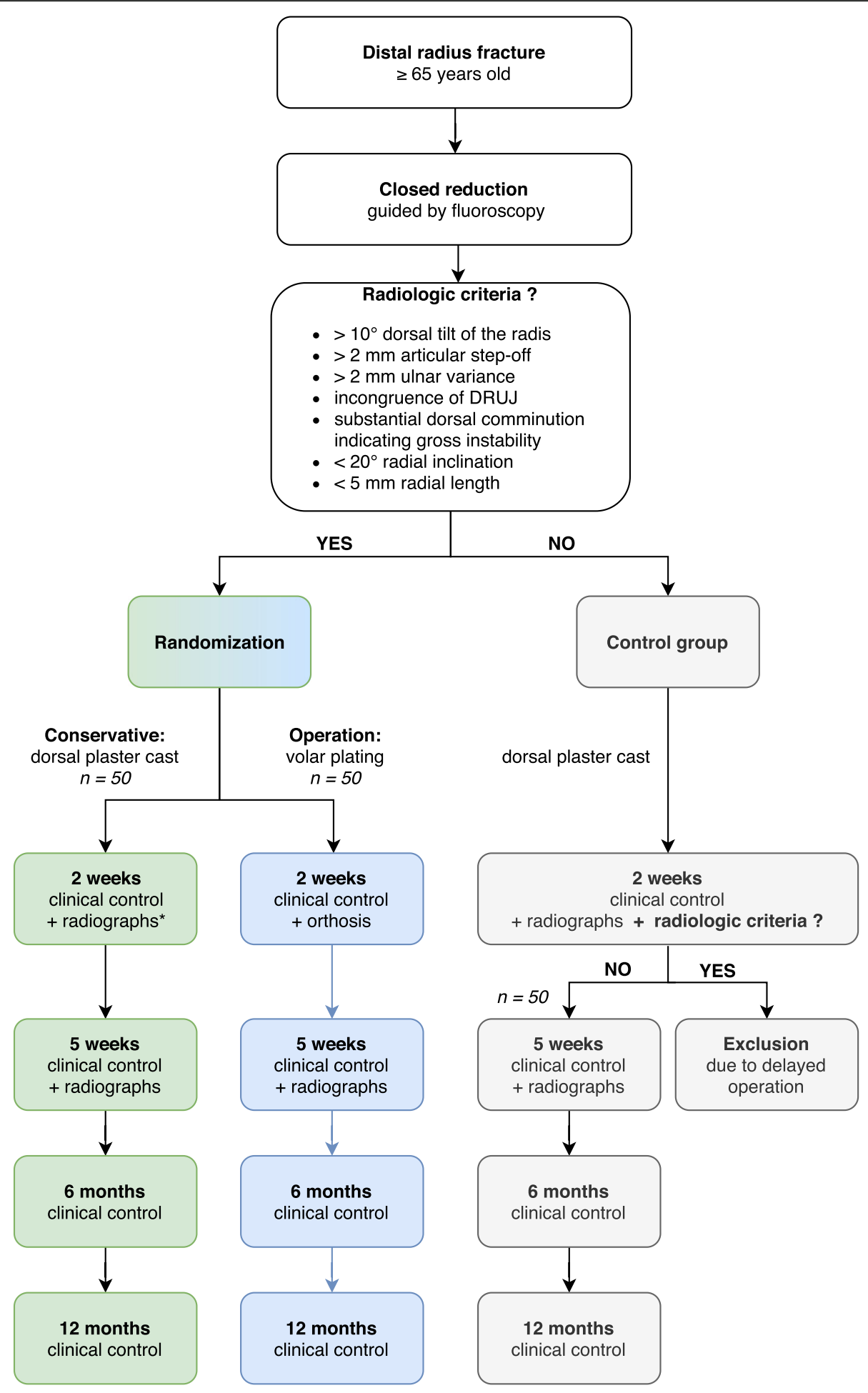

Fig. 1 Study Design. An overview of the study design showing the 3 arms. *These radiographs are evaluated respectively after study completion and do not influent the prospective follow-up of the individual patient 
Arm 2: closed reduction and 5-weeks dorsal plaster cast immobilization with a single hand therapeutic instruction;

Arm 3: a control group of patients with minimally displaced DRF that do not fulfill the radiologic criteria.

Follow-up time is planned to be 1 year with out-patient visits at 2 weeks, 5 weeks, 6 months and 12 months after the injury. This trial is approved by the Danish Scientific Ethical Committee on the 3rd of September 2018. This study is carried out at the Regional Hospital Randers, Denmark with a coverage area of approximately 270,000 inhabitants.

\section{Eligibility criteria}

All patients with DRF diagnosed at the emergency department are screened for eligibility.

\section{Intervention groups: (arm 1 and arm 2)}

Eligibility criteria for participants who will be allocated to random treatment are:

$-\geq 65$ years old

- low-energy distal radius fracture.

The distal radius fracture must fulfill at least one of the following radiological criteria after closed reduction in the emergency department in order to be randomized between treatment arm 1 and 2:

$->10^{\circ}$ dorsal tilt of the radius in relation to perpendicular to the longitudinal axis of radius

- >2 mm Ulnar variance

- $>2 \mathrm{~mm}$ Articular step-off

- Incongruence of the distal radioulnar joint

- Substantial dorsal comminution

$-<20^{\circ}$ Radial inclination

- $<5$ mm Radial length

\section{Control group: (arm 3)}

Eligibility criteria for participants in the control group, arm 3:

$-\geq 65$ years old

- low-energy distal radius fracture.

This distal radius fracture had to fulfill all the following radiologic criteria:

$-\leq 10^{\circ}$ Dorsal tilt of the radius in relation to perpendicular to the longitudinal axis of radius

$-\leq 2 \mathrm{~mm}$ Ulnar variance

- $\leq 2 \mathrm{~mm}$ Articular step-off

- No incongruence of the distal radioulnar joint $-\geq 20^{\circ}$ Radial inclination

- $\geq 5 \mathrm{~mm}$ Radial length

\section{Exclusion criteria}

- Patients $<65$ years

- high-energy fracture

- open fracture

- concomitant injuries, e.g. multiple fractures on afflicted arm

- not capable of giving written consent

- previous DRF or forearm fracture on the same side

\section{Recruitment}

Any participant must be approved eligible for the study by either one of the consultants in the research group or the house physician on call. Patients are primarily recruited by directly contact in the emergency room on the day of primary contact, where they are informed about the study and asked for written consent. The Danish consent form and patient information material is given to the patient, a blank sample can be ordered from the corresponding author. Every patient who is treated in the emergency department during a shift is discussed the following day on a conference, where all radiographs also are reviewed. This additional control ensures that every potential participant is assed for eligibility and offered enrollment in the study either directly in the emergency room or the day after by telephone. When recruitment is done over the telephone, written consent is obtained before surgery or at the 2 week out-patient visit, if the participant should be randomly assigned to conservative treatment or if the patient is in the control group. The recruiting health care personnel randomly assigns participants to the interventions as described below.

\section{Randomization}

Randomization is executed by random drawing of sealed, opaque envelopes. According to the sample size calculation, 50 participants will be allocated to each group, hence 100 identical A5 envelopes have been sealed - each containing a folded note whereupon either "operative" or "conservative" is written. In order to assure similar timewise enrolment the following measures will be applied (Fig. 2). The 50 envelopes for operative treatment will be packed into stacks of 5 envelopes. The same will be done with the 50 envelopes for conservative treatment. One stack of each treatment arm will be mixed resulting in an equal chance to draw either treatment or intervention among the ten envelopes. The including health care personnel will draw one of the 10 envelopes and hence allocate the participant randomly to either treatment arm 1 or 2 . When there are only three envelopes left, one stack of each group will be opened and mixed into the 

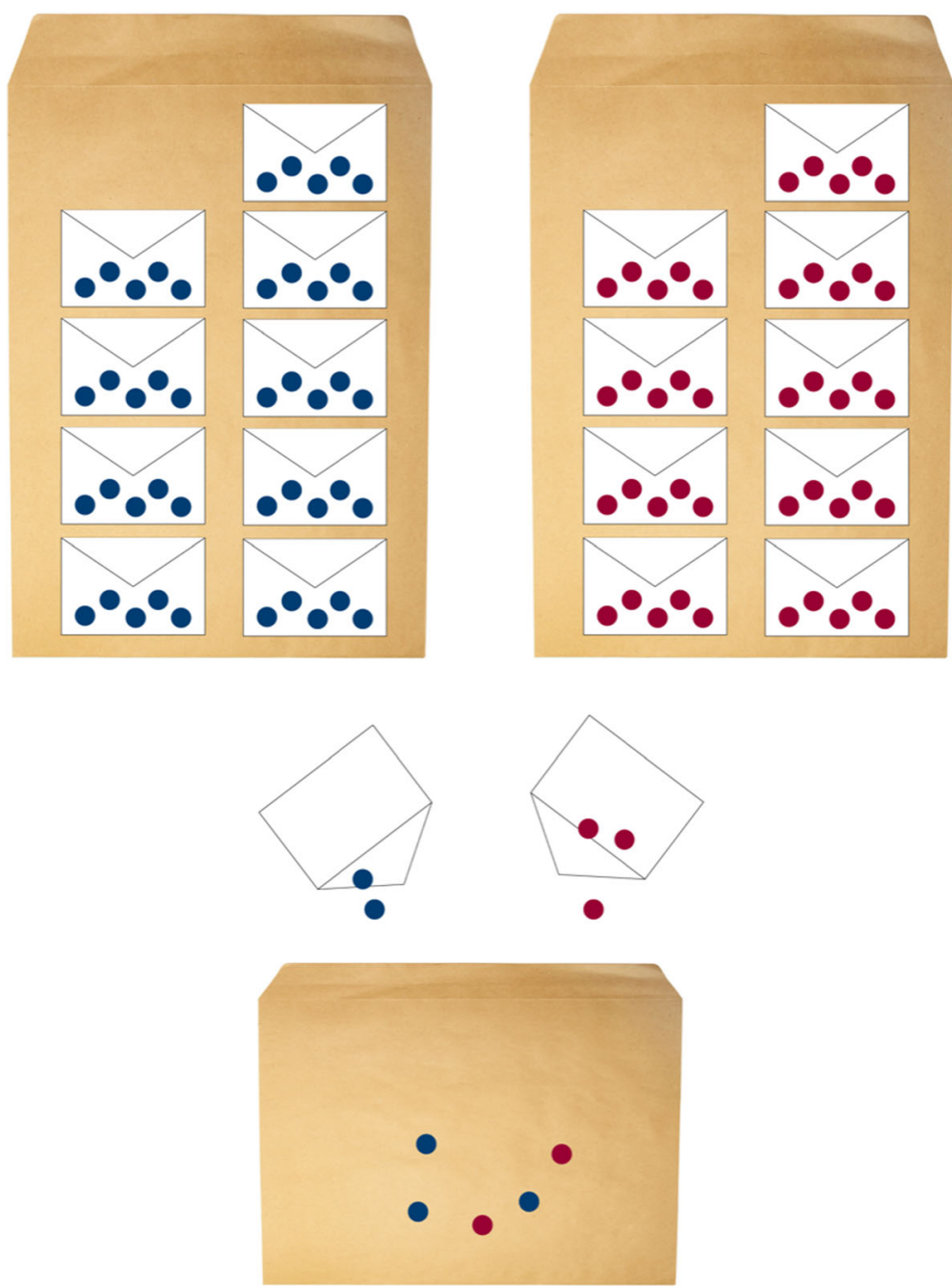

Fig. 2 Illustration of the randomisation process regarding intervention groups, e.g. treatment arm 1 and 2. Each dot represents a sealed, opaque envelope that contains a note with the treatment arm allocation. The blue and red dots represent "conservative" and "operative" treatment, respectively. Whenever only 3 envelopes are left, 5 new "operative" and 5 new "conservative" treatment envelopes are mixed and added

remaining. By this measure, the health care personnel cannot predict the allocated treatment based upon the order of previous mixed treatment allocation from the mixed pool of small envelopes.

\section{Interventions}

Interventions when a possible participant addresses the emergency room

After diagnosing the fracture on a standardized wrist radiograph (anterior-posterior projection and lateral projection) in the emergency room, the physician on call has two attempts to achieve an acceptable closed reduction under local analgesia with a $20 \mathrm{mg} / \mathrm{ml}$ Lidocaine hematoma block. While the effect of the hematoma block sets in, nurses measure the arm to be able to lay a proper dorsal plaster cast immobilization.
Fluoroscopy is readily available in the emergency room and guides the closed reduction and plaster immobilization. After reduction standardized radiographs are obtained at the department of radiology and the quality of the closed reduction is assessed by the physician on duty. If the radiologic eligibility criteria are fulfilled after closed reduction, the patient is informed about the study and offered enrollment. If the fracture is less severe, e.g. without any of the radiologic criteria warranting closed reduction or operation as mentioned above, the wrist is immobilized with a plaster cast without closed reduction.

\section{Intervention group}

Treatment arm 1: 
Open reduction and volar plate fixation utilizing Acu-Loc ${ }^{\circ}$, Acumed or Variax ${ }^{\circ}$, Stryker with a standard Henry approach to the distal radius and pronator quadratus repair if possible. The vast majority of patients will be operated in regional anesthesia and the remaining patients in general anesthesia. It is the choice of the surgeon whether a tourniquet will be used. After surgery the wrist is immobilized in a dorsal plaster cast for 2 weeks followed by further 3 weeks of immobilization with a removable orthosis. A single hand therapeutic instruction will take place.

Treatment arm 2:

Conservative treatment consists of dorsal plaster cast immobilization for 5 weeks. Only discomfort, neurologic deficits or signs of infection warrant removal and replacement with another dorsal plaster cast. A single hand therapeutic instruction will take place.

\section{Control group}

Patients with less displaced fractures, before or after and eventual closed reduction will be:

Conservatively treated as described for treatment arm

2.

Patients in the control group that fulfill the radiologic criteria after 2 weeks due to loss of reduction, are offered operative treatment according to the NCG and hence will be excluded from the trial.

The investigators reserve the right to exclude a participant if it is considered clinically irresponsible to let them continue.

\section{Outcomes}

Summarized in Table 1.

\section{Primary outcomes}

The complication rate will be estimated at day 0,2 weeks, 5 weeks, 6 months, and 12 months after the injury.

Complications are defined as the presence of:

- Sensory disturbance, including carpal tunnel syndrome and chronic regional pain syndrome

- Flexor tendon rupture and irritation

- Extensor tendon rupture and irritation

- Hardware failure, e.g. osteosynthesis loosening

- Infection: superficial

- Infection: deep

- Reoperation with hardware replacement

- Reoperation with hardware removal (partial or total), which is not routinely performed in Denmark

- Vascular compromised (capillary refill $\geq 2 \mathrm{~s}$ )

Patients will report complications at the given timepoints by answering a questionnaire stating either yes / no and a free-text explanation. If the patient states any complications, a member of the research group will qualify the

Table 1 Illustration of timeline and outcome measures including baseline demographics

\begin{tabular}{|c|c|c|c|c|c|}
\hline & DRF & 2 weeks & 5 weeks & 6 months & 12 months \\
\hline \multicolumn{6}{|c|}{ Primary outcome complications } \\
\hline Questionaire & $x$ & $x$ & $x$ & $x$ & $x$ \\
\hline Examination & $x$ & $x$ & $x$ & $x$ & $x$ \\
\hline \multicolumn{6}{|l|}{ Secondary outcome } \\
\hline \multicolumn{6}{|l|}{ Patient reported outcome } \\
\hline Quick DASH (DK) & $x$ & $x$ & $x$ & $x$ & $x$ \\
\hline PRWHE (DK) & & & & $x$ & $x$ \\
\hline EQ5D & & & & $x$ & $x$ \\
\hline Pain at rest $(0-10)$ & $x$ & $x$ & $x$ & $x$ & $x$ \\
\hline \multicolumn{6}{|l|}{ Objective examination } \\
\hline Wrist range of motion & & & $x$ & $x$ & $x$ \\
\hline Grip strength & & & & $x$ & $x$ \\
\hline Pinch gauge & & & & $x$ & $x$ \\
\hline Wrist radiographs & $x$ & $x$ & $x$ & & \\
\hline
\end{tabular}

Baseline demographics:

Age, gender, hand dominance, working status, ASA class, diabetes, smoking, alcohol consumption

Illustration of timeline and outcome measures including baseline demographics Abbreviations: Quick DASH (DK) The quick disability of the arm, shoulder and hand outcome measure - validated Danish translation, PRWHE (DK) Patient-related wrist evaluation score - validated Danish translation, ASA class American Society of Anesthesiologists Classification 
answer and fill in the free text. However, a YES can only be qualified and shall never be erased if the physician does not agree with the patient's opinion or explanation.

The Danish version of the Quick Disabilities of the Arm, Shoulder and Hand [22] will be used to assess the level of functionality prior to injury, after 2 weeks, 5 weeks, 6 months, and 12 months. The minimal clinically relevant difference is 16 to 20-point difference in Quick DASH [19-21].

Range of motion is measured by a registered nurse using a goniometer. To ensure the observer is blinded, the patient is instructed not to talk about the treatment. Furthermore, all wrists will be covered by a glove concealing potential scares (Fig. 3). The following data are thus collected in a blinded fashion wrist flexion, extension, pronation, supination, radial deviation, ulnar deviation. The contralateral side will serve as a reference and history of injuries or operations of the contralateral side will be recorded.

\section{Secondary outcomes}

The patient reported outcome measure Quick DASH will be supported by the following secondary outcome measures:

- A Danish version of the Patient rated Wrist Evaluation questionnaire (PRWE) will be evaluated after 6 month and 12 months [23].

- Grip strength of both left and right hand will be estimated as the maximum and average of score of three repetitions of each hand with alternating hands between attempts after 6 months and 12 months using a dynamometer.

- A pinch gauge where both left and right hand are evaluated (yes/no) if the participant can pinch a sheet of paper. This is collected after
6 months and 12 months by an unblinded physician or research year student.

- The potential flexion deficit of the 1st finger towards the base of the 5th finger measured as the distance $(\mathrm{cm})$ from the pulp of the 1st finger to the carpometacarpal joint of the 5th finger after 6 and 12 months.

- The pulp-to-palm distance of the distal 2nd-5th finger and palmar surface of the side treated for DRF after 6 and 12 months.

- The experienced pain during activity within the preceding 14 days before the injury and at 6 - and 12-months follow-up stated on a $0-10$ Numeric Rating Scale (NRS).

- The pain at rest after 2 and 5 weeks given NRS.

- EQ5D (European Quality of life - 5 Dimensions) after 6 months and 12 months. This is registered by an unblinded physician or research year student [24]. - The self-reported use of pain medication at day 0 , after 2 weeks, 5 weeks, 6 months, and 12 months.

- The prescribed use of pain medication compared 12 months before with 3 and 12 months after the injury.

- Standardized radiographs of the injured wrist at day 0 before and after closed reduction (all groups), week 2 (conservative treatment group, not reviewed before completion of the follow-up period), week 5 (all groups).

The following baseline demographics will be recorded: gender, age, side of DRF, hand dominance (right-handed, left-handed, ambidextrous), working status, American Society of Anesthesiologists Classification (ASA class 1-6), smoking (cigarettes/day), alcohol consumption (units/week) and diabetes (yes/no).

\section{Blinding}

The study is single blinded, as all measurements will be performed with a glove masking a potential scare. Hence,

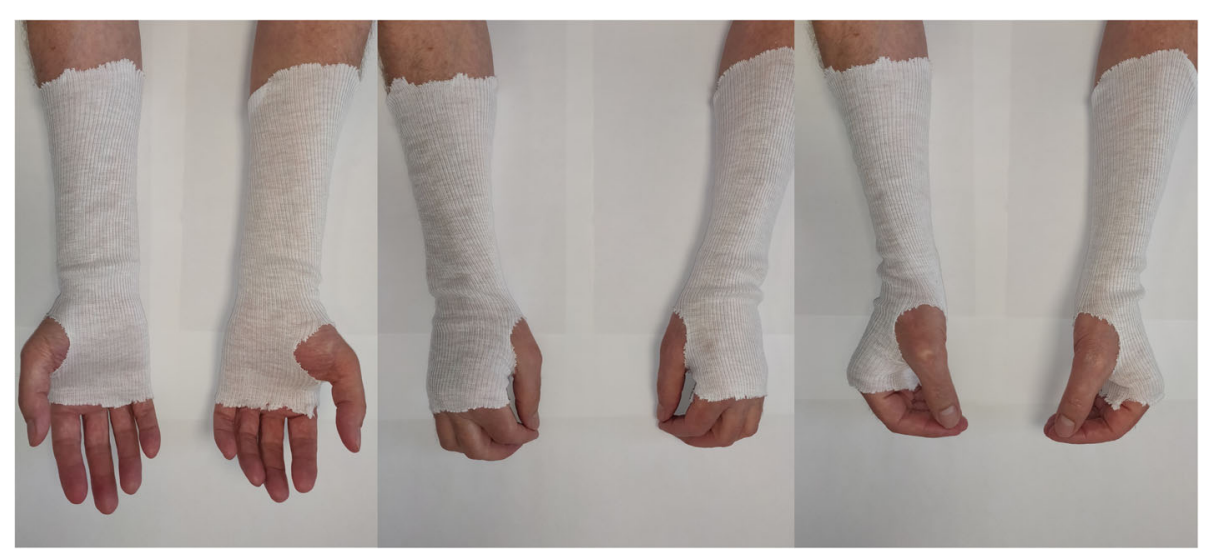

Fig. 3 A demonstration of the blinding of treatment using a glove 
the observer will be blinded when examining the participant. Furthermore, before each visit the patient is instructed, not to talk about the received treatment with the observer.

During the planning of the study, sham-operations were taken in consideration in order to blind the participants. However, most DRF are operated wide-awake under regional anesthesia only. The research group considered performing a skin incision and writing a manuscript simulating all the noises and communication, that usually take place during an operation. However, the efficacy of this potential blinding of participants during wide-awake surgery was deemed questionable. General anesthesia would have been a viable option, but it would vary too much from the current practice to be feasible at our hospital.

\section{Surgeon experience and type of plates}

Our previous retrospective follow-up study concluded, that neither surgeon experience nor type of volar locking plate was associated with the complication rate [3]. Therefore, we consider surgeon experience and type of plate of no to minor clinical importance for the outcomes of this study. Hence, all physicians that usually treat DRF conservatively and operatively at our hospital will treat patients. No selection, nor restrictions regarding treating physician and fracture type will be imposed and operating physicians range from residents to consultants.

\section{Statistical plan and analysis Sample size}

The sample size was calculated based on a $20 \%$ difference in complication rate between the two treatment groups, an alpha level of $5 \%$ and a power of $80 \%$. Consequently, each group shall at least consist of 49 participants. The control group was decided to be of equal size.

\section{Data management}

All data will be managed in accordance with Good Clinical Practice. Papers containing patient identifiable data along with informed consent are physically stored in a locked room. Study data will be collected and managed using REDCap electronic data capture tools hosted at Aarhus University, Denmark [25]. The Data Steering Committee (RT, JDR, JP) will review included and excluded patients every 14th day. If patients do not show up for follow-up in the outpatient clinic, The Data Steering Committee will contact the patient by phone and/or mail in order to ensure participant retention and complete follow-up. Only the Data Steering Committee will have access to the final trial data set. No publication of the data is planned; however data will be stored according to national legislation.

\section{Statistical analysis plan}

That data will be analyzed using Fisher's exact test and Mann Whitney U test. The desired applied statistic is odds ratio with Pearson's 95\% confidence interval. Should any data be lost in the follow-up, the Last Observation Carried Forward concept will be used. Treatment arm 3 will be analyzed after 6 months follow-up and published separately.

All test will be two-tailed and assessed at the 5\% alpha level. Categorical measures will be presented as percentages. An intention-to treat and per-protocol analysis will be considered. Continuous measures will be presented as means with standard deviations and medians with inter-quartile range. Treatment effects over time will be assessed using linear mixed effect models with patient treated as random factor. A normal distribution with an identity link function will be assumed for continuous measures, while a multinomial distribution and cumulative logit function will be applied to ordinal outcomes.

\section{Discussion}

This prospective trial helps to clarify the best treatment strategy for displaced DRF patients $\geq 65$ years. Practical limitations prevent the conduction of a double-blinded data collection, as no sham operations will be performed.

To the best of our knowledge, only one similar randomized controlled trial investigating volar locking plates versus conservative treatment in patients $\geq 65$ years with DRF has been conducted $[8,16]$. In 2011, Arora and coworkers reported similar results in terms of patientreported outcome measures DASH and PRWE, pain level and range of motion between 36 operatively and 37 nonoperatively treated patients [16], while the complication rate was 36 and $14 \%$ in the two groups, respectively. However, this trial did not have a significant clinical impact on the treatment of this patient group in Denmark. In the light of these promising initial report limiting the need of surgical intervention, there is a need to verify its results. Thus, the current trial will help to clarify the best treatment strategy for displaced DRF in patients $\geq 65$ years in terms of complication rate and expected comparable functional outcome. If the results of the study indicate one treatment superior to the other, clinical guidelines are likely to be influenced by the current study.

Furthermore, cost effectiveness calculations can be performed on the basis of results of the current study and unnecessary operations may be prevented in order to live up to the Hippocratic oath, "primum non nocere - first do no harm'.

\section{Abbreviations}

ASA class: American Society of Anesthesiologists Classification; DRF: Distal radius fractures; EQ5D: European Quality of life - 5 dimensions; 
NCG: National Clinical Guidelines; NRS: Numeric Rating Scale; ORIF: Open reduction internal fixation; PRWE: Patient rated Wrist evaluation; Quick DASH: Quick Disabilities of the Arm, Shoulder and Hand

\section{Acknowledgements}

Not applicable.

\section{Dissemination of findings}

Regardless of the findings, results will be submitted for peer-reviewed publication. Furthermore, results will be prepared for presentation on relevant conferences. Authorship will be granted according to International Committee of Medical Journal Editors guidelines.

\section{Authors' contributions}

All authors contributed to the current protocol: conception and design (JP, $J D R, R T)$, acquisition of data (JP, RT), drafting of the manuscript and critical revision (JP, SOM, JDR, RT), statistical analysis (JDR, RT), analysis and interpretation of data (JP, JDR, RT), obtaining funding (JP, RT) and supervision (JDR, RT). All authors read and approved the final manuscript.

\section{Funding}

Expenses through salary specified by Aarhus University to the research year student is covered externally by The Research Fund of Randers Regional Hospital and the Department of Orthopedics at Regional Hospital Randers has provided deficit guarantee. Expenses to nurses for compensation of extra work load is externally covered by The Research Fund of Randers Regional Hospital. All expenses are financed by regional public health care budget. No specific funding has been received for this study.

\section{Availability of data and materials}

Not applicable.

\section{Ethics approval and consent to participate}

This study has been approved by the Danish Scientific Ethical Committee (ID: 1-10-72-420-17) and has been registered at the Danish Data Protection Agency. Protocol modifications are prohibited. Furthermore, this study is registered at Clinicaltrials.gov (ID: NCT03716661). Participation is based on written consent after the possible participant is carefully informed about participation by the including physician or research year student. Only information regarding the therapeutic process of the wrist and associated hospitalizations within the 1 year of follow-up will be extracted from the participants electronic medical journals. The surgical treatment is covered by The Danish Patient Compensation Association.

We do not expect that participants experience more and severe complications than those associated to the standard operative treatment. Plaster immobilization may lead to complications e.g. skin irritation, pain, and at rare occasions ulcer due to poorly applied plaster. All patients are given instructions for cast complications. Normally, this can be fixed by replacing the plaster cast. As previously stated, the investigators reserve the right to exclude participants at any time if it is considered clinically irresponsible to let them continue in the study. An interim analysis of the 6 months follow-up data will be performed when half of the patients has reached that milestone. The study will be terminated if treatment arm 2 should be inferior to treatment arm 1 in terms of complication rate. The patient can anytime withdraw their written consent.

\section{Consent for publication}

Written consent for publishing has been obtained by the individual in the images for Fig. 3.

\section{Competing interests}

RT is consultant for Acumed. The other authors have no conflict of interest.

\section{Author details}

'Department of Orthopedics, Regional Hospital Randers, Skovlyvej 15, DK-8930 Randers, Denmark. ${ }^{2}$ Department of Orthopedics, Aarhus University Hospital, Aarhus, Denmark. ${ }^{3}$ Institute of Clinical Medicine, Aarhus University, Aarhus, Denmark.
Received: 8 April 2019 Accepted: 11 June 2019

Published online: 29 June 2019

\section{References}

1. Baron JA. Basic epidemiology of fractures of the upper and lower limb among Americans over 65 years of age. Epidemiology (Cambridge, Mass). 1996;7(6):612-8

2. Christensen M. Treatment of distal radius fractures in the elderly. Ugeskr Laeger. 2015;177(34). https://www.ncbi.nlm.nih.gov/pubmed/26320591.

3. Thorninger R. Complications of volar locking plating of distal radius fractures in 576 patients with 3.2 years follow-up. Injury. 2017:48(6):1104-9.

4. Brogren E. Incidence and characteristics of distal radius fractures in a southern Swedish region. BMC Musculoskelet Disord. 2007;8(1):48.

5. Flinkkilä T. Epidemiology and seasonal variation of distal radius fractures in Oulu, Finland. Osteoporos Int. 2011;22(8):2307-12.

6. Hernlund E, Svedbom A, Ivergard M, Compston J, Cooper C, Stenmark J, et al. Osteoporosis in the European Union: medical management, epidemiology and economic burden. A report prepared in collaboration with the International Osteoporosis Foundation (IOF) and the European Federation of Pharmaceutical Industry Associations (EFPIA). Arch Osteoporos. 2013:8:136.

7. Statistics Denmark Population Projection 2018-2060; 27 Mar 2019. Available from: https://www.dst.dk/da/Statistik/nyt/NytHtml?cid=26827.

8. Sundhedssyrelsen. Nationale Kliniske Retningslinjer for behandling af håndledsnære brud (distal radius fraktur) 201427 Mar 2019. Available from: https:/www.sst.dk/da/udgivelser/2014/ /media/ EB0328BE70DE4D4AB5C6E164EC87C3BA.ashx?m=.pdf.

9. Madsen ML, Waever D, Borris LC, Nagel LL, Henriksen $M$, Thorninger $R$, et al. Volar plating of distal radius fractures does not restore the anatomy. Dan Med J. 2018;(8):65. https://www.ncbi.nlm.nih.gov/pubmed/30059004.

10. Wichlas F, Haas NP, Disch A, Macho D, Tsitsilonis S. Complication rates and reduction potential of palmar versus dorsal locking plate osteosynthesis for the treatment of distal radius fractures. J Orthop Traumatol. 2014;15(4):259-64.

11. Arora R, Lutz M, Hennerbichler A, Krappinger D, Espen D, Gabl M. Complications following internal fixation of unstable distal radius fracture with a palmar locking-plate. J Orthop Trauma. 2007;21(5):316-22.

12. Williksen JH, Frihagen F, Hellund JC, Kvernmo HD, Husby T. Volar locking plates versus external fixation and adjuvant pin fixation in unstable distal radius fractures: a randomized, controlled study. J Hand Surg. 2013;38(8): 1469-76.

13. Rampoldi M, Marsico S. Complications of volar plating of distal radius fractures. Acta Orthop Belg. 2007:73(6):714-9.

14. Snoddy MC, An TJ, Hooe BS, Kay HF, Lee DH, Pappas ND. Incidence and reasons for hardware removal following operative fixation of distal radius fractures. J Hand Surg. 2015;40(3):505-7.

15. Shauver MJ. An economic analysis of outcomes and complications of treating distal radius fractures in the elderly. J Hand Surg (American ed). 2011;36(12):1912-8.e3.

16. Arora R. A prospective randomized trial comparing nonoperative treatment with volar locking plate fixation for displaced and unstable distal radial fractures in patients sixty-five years of age and older. J Bone Joint Surg (Am Vol). 2011;93(23):2146-53.

17. Lutz K. Complications associated with operative versus nonsurgical treatment of distal radius fractures in patients aged 65 years and older. J Hand Surg (American ed). 2014:39(7):1280-6.

18. van Leerdam RH, Huizing F, Termaat F, Kleinveld S, Rhemrev SJ, Krijnen P, et al. Patient-reported outcomes after a distal radius fracture in adults: a 3-4 years follow-up. Acta Orthop. 2019;90(2)129-134. https://doi.org/10.1080/ 17453674.2019.1568098.

19. Franchignoni F, Vercelli S, Giordano A, Sartorio F, Bravini E, Ferriero G. Minimal clinically important difference of the disabilities of the arm, shoulder and hand outcome measure (DASH) and its shortened version (QuickDASH). J Orthop Sports Phys Ther. 2014;44(1):30-9.

20. Mintken PE, Glynn P, Cleland JA. Psychometric properties of the shortened disabilities of the arm, shoulder, and hand questionnaire (QuickDASH) and numeric pain rating scale in patients with shoulder pain. J Shoulder Elb Surg. 2009;18(6):920-6.

21. The DASH Outcome Measure - Disabilities of the Arm, Shoulder, and Hand Available from: http://www.dash.iwh.on.ca/faq. Accessed 26 June 2019. 
22. Schonnemann JO, Eggers J. Validation of the Danish version of the quick-disabilities of arm, shoulder and hand questionnaire. Dan Med J. 2016;63(12). https://www.ncbi.nlm.nih.gov/pubmed/?term=27910799.

23. Hansen $A \varnothing$, Knygsand-Roenhoej K, Ardensø K. Danish version of the patient-rated wrist/hand evaluation questionnaire: translation, crosscultural adaptation, test-retest reliability and construct validity. Hand Ther. 2019;24(1):22-30.

24. Wittrup-Jensen KU, Lauridsen J, Gudex C, Pedersen KM. Generation of a Danish $T$ O value set for EQ-5D health states. Scand J Public Health. 2009;37(5):459-66.

25. Harris PA, Taylor R, Thielke R, Payne J, Gonzalez N, Conde JG. Research electronic data capture (REDCap)--a metadata-driven methodology and workflow process for providing translational research informatics support. J Biomed Inform. 2009;42(2):377-81.

\section{Publisher's Note}

Springer Nature remains neutral with regard to jurisdictional claims in published maps and institutional affiliations.

Ready to submit your research? Choose BMC and benefit from:

- fast, convenient online submission

- thorough peer review by experienced researchers in your field

- rapid publication on acceptance

- support for research data, including large and complex data types

- gold Open Access which fosters wider collaboration and increased citations

- maximum visibility for your research: over $100 \mathrm{M}$ website views per year

At BMC, research is always in progress.

Learn more biomedcentral.com/submissions 